\title{
El sector demoscópico en un nuevo escenario: retos y particularidades
} New Scenery to Demoscopic sector: Challenges and Peculiarities

\author{
José Juan García Vázquez \\ GAD3 \\ josejuan.garcia@gad3.com \\ Recibido / Received: 14/12/2016 \\ Aceptado / Accepted: 07/03/2017
}

\section{RESUMEN}

El paso del bipartidismo de concentrar tres de cada cuatro votos a obtener poco más de uno de cada dos en una sola legislatura ha supuesto quitar el suelo sobre el que han trabajo los sondeos pre-electorales durante los anteriores treinta años. De un votante estable, se pasa a un votante cambiante y dubitativo. De un marco formado por un único eje - derecha/ izquierda - a un nuevo marco en el que se cruzan varias dimensiones. El escenario para la realización de estimaciones es otro, el juego es también otro. Sin embargo, la actividad demoscópica española sólo conoce las reglas de un juego que ya no está en el mercado. Como ocurre con el mercado de los videojuegos, la nueva edición ya no vale para el modelo de máquina anterior, por lo que se exige adquirir un nuevo aparato: una nueva metodología para la estimación.

Palabras clave: Abstención electoral, sistema de partidos, redes sociales, big data.

\section{ABSTRACT}

The fast change of bipartisanship from concentrating three out of four votes to get little more than one in two in a single legislature has meant removing the ground on which pre-electoral polls have worked during the previous thirty years. Ground that passes from a stable to a changing and hesitant voter. From a frame formed by a single axis — right/left — to a new frame in which several dimensions cross. The scenery for making estimates is another, the game is also another. However, Spanish demoscopic activity only knows the rules of a game that is no longer on the market. As with the videogame market, the new edition no longer applies to the previous machine model, so a new device is required: a new methodology for estimating.

Key words: Electoral absenteeism, party system, social networks, big data.

*Autor para correspondencia / Corresponding author: José Juan García Vázquez. GAD3. c./ Alcalá, 75-4. ${ }^{\circ}$ Izquierda, 28009 Madrid, España/Spain. Sugerencia de cita / Suggested citation: García Vázquez, J. J. (2017). El sector demoscópico en un nuevo escenario: retos y particularidades. Revista Española de Sociología, 26 (3 Supl.), 147-155.

(http://dx.doi.org/10.22325/fes/res.2017.15) 


\section{INTRODUCCIÓN}

El periodo 2015-2016 fue una etapa cargada de citas electorales en España. En poco más de un año, se sucedieron en nuestro país numerosos procesos electorales: Elecciones Andaluzas (marzo de 2015), Municipales y Autonómicas (mayo de 2015), Elecciones Catalanas (septiembre de 2015) y dos Elecciones Generales (diciembre de 2015 y junio de 2016). A raíz del clima incierto y menos previsible que años atrás, las encuestas se convirtieron en un elemento de referencia para conocer la voluble realidad política ${ }^{1}$. Además, el XII Congreso se celebró el 30 de junio y 1-2 de julio, pocos días después de los comicios del 26 de junio, con el foco mediático centrado en el sector demoscópico, al que de manera implícita se le exigía una rendición de cuentas.

Pero, en mi opinión, aún más acertada es la clausura de mano del sector demoscópico por su ajuste al lema del Congreso "Grandes transformaciones sociales, nuevos desafíos para la Sociología". Estas transformaciones sociales aludidas, son aún más pronunciadas en esta área, que ha visto como la realidad política española ha cambiado sustancialmente en los últimos años. La realidad se impone. Y ha sido esta realidad la que nos ha llevado a replantearnos la metodología establecida y su vigencia. En este nuestro campo, más que nunca, el sociólogo debe vivir pegado a la realidad para calibrar las nuevas tendencias en lo que, sin duda, supone un desafío constante.

Aprovechando la pertinencia del lema del Congreso para la etapa que vive nuestro sector, el presente artículo se jalona en estos dos apartados: transformaciones sociales y nuevos desafíos para la Sociología en lo que, simplificando,

1 Por cuantificar esta afirmación, se puede recurrir a la Memoria de Actividades 2015 del Centro de Investigaciones Sociológicas donde se puede consultar el acceso a su Banco de Datos. Claramente, la publicación de estudios políticos (Barómetros de enero, abril, julio y octubre y estudios pre y post-electorales) marcan las mayores cotas de demanda de datos: (CIS, 2016). podemos identificar como la realidad social y su medición.

En primera instancia, se describen y analizan los principales cambios políticos y electorales acontecidos en España en los últimos años. A continuación, se aborda el esfuerzo de la demoscopia para incorporar estos cambios y continuar manteniendo su capacidad explicativa, incidiendo en los retos metodológicos y las estrategias de análisis. En última instancia, se reflexiona sobre las particularidades del sector demoscópico dentro de la disciplina sociológica.

\section{GRANDES TRANSFORMACIONES SOCIALES}

La Sociología asume la difícil tarea de realizar ciencia sobre un objeto de estudio en permanente cambio. Además, en las sociedades occidentales, este cambio cada vez se produce de forma más acelerada (Rosa, 2011). Y España no es una excepción. Centrando el enfoque en el análisis electoral que nos ocupa, nuestro país ha experimentado numerosos cambios de gran calado que han configurado un escenario muy diferente al de anteriores legislaturas. En primer lugar, cabe destacar la aparición de dos nuevos partidos que se estrenan en el Congreso con 69 (Podemos y sus confluencias) y 40 diputados (Ciudadanos), convirtiéndose en actores políticos indispensables para la formación de gobierno. Estos partidos surgen porque se han producido cambios sociales que cimentan su entrada en el sistema político. Sin las condiciones propicias, ni habrían surgido, ni se habrían consolidado. № obstante, en este breve artículo la descripción de las transformaciones se restringe al ámbito político que nos ocupa.

La irrupción de estos nuevos partidos hace retroceder al bipartidismo hasta su cota más baja y configura el Parlamento con el índice de fragmentación más alto de la historia democrática reciente: 0,78 en la XI Legislatura (Figura 1). En los comicios de 2011 , el $73 \%$ de los votos válidos correspondieron a PP o PSOE, en 2015 sólo uno de cada dos. En escaños, los dos principales partidos perdieron 83 diputados en el transcurso de esta legislatura (Tabla 1). 


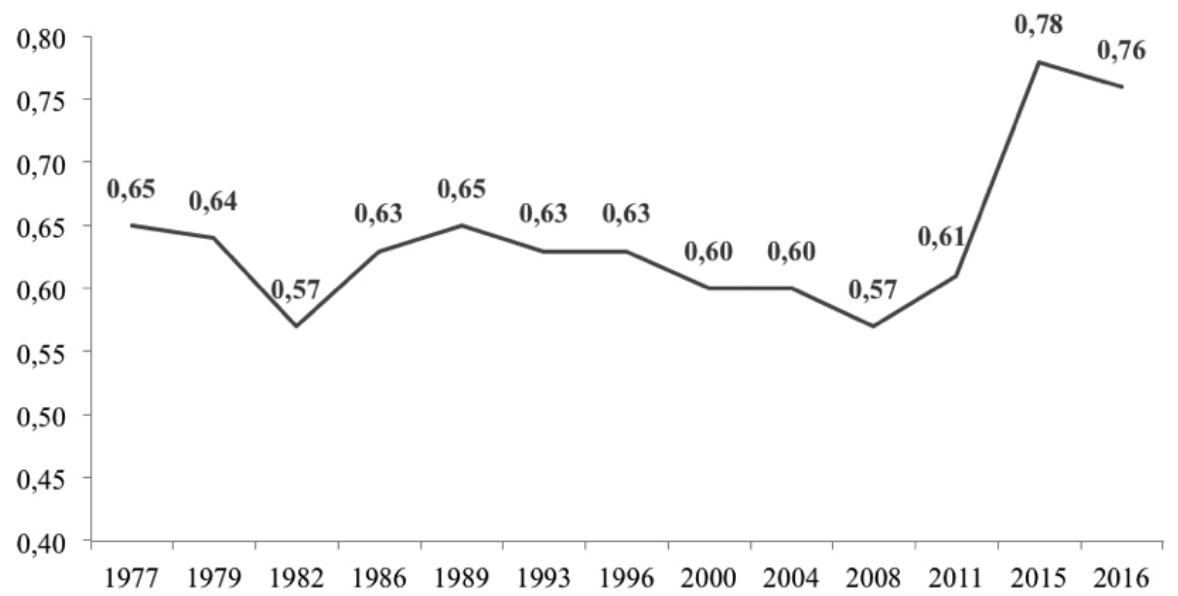

Figura 1. Índice de Fragmentación Parlamentaria por legislatura. España (1977-2016)²

Fuente: Elaboración propia a partir de datos del Ministerio del Interior. http://www.infoelectoral.interior.es/

Tabla 1. Índice de Concentración Electoral de los dos partidos más votados en Elecciones Generales. España (1977-2016)

\begin{tabular}{c|c|c|c|c}
\hline Elección & Principales Partidos & N. ${ }^{0}$ votos & \% Voto válido & Escaños \\
\hline 1977 & UCD+PSOE & 11682257 & $63,8 \%$ & 283 \\
\hline 1979 & UCD+PSOE & 11738406 & $65,2 \%$ & 289 \\
\hline 1982 & PSOE+AP & 15675499 & $74,5 \%$ & 309 \\
\hline 1986 & PSOE+AP & 14149395 & $70,0 \%$ & 289 \\
\hline 1989 & PSOE+PP & 13401540 & $65,4 \%$ & 282 \\
\hline 1993 & PSOE+PP & 17351546 & $73,5 \%$ & 300 \\
\hline 1996 & PP+PSOE & 19141684 & $76,4 \%$ & 297 \\
\hline 2000 & PP+PSOE & 18239930 & $78,7 \%$ & 308 \\
\hline 2004 & PSOE+PP & 20789307 & $80,3 \%$ & 312 \\
\hline 2008 & PSOE+PP & 21567345 & $83,8 \%$ & 323 \\
\hline 2011 & PP+PSOE & 17870077 & $73,4 \%$ & 296 \\
\hline 2015 & PP+PSOE & 12782280 & $50,7 \%$ & 213 \\
\hline 2016 & PP+PSOE & 13385082 & $55,6 \%$ & 222 \\
\hline
\end{tabular}

Fuente: Elaboración propia a partir de datos del Ministerio del Interior. http://www.infoelectoral.interior.es/

2 El índice de fragmentación parlamentaria, cuyo valor oscila entre 0 (sólo un partido en el Parlamento) y 1 (hay tantos partidos como diputados), es la diferencia de la unidad menos la suma de los cuadrados de la proporción de escaños de cada candidatura con representación parlamentaria. 
El nuevo escenario complica la metodología demoscópica contrastada durante décadas. El sistema partidista deja de asemejarse a un juego de suma cero con todas las variables controladas y el trasvase de votos entre partidos/abstención ya no está tan claro. De hecho, para interpretar esta nueva realidad no sólo sirve la lectura en el eje izquierda/derecha, sino que es preciso incorporar la dimensión partidos nuevos/partidos tradicionales, entre otras muchas variables referidas al electorado. Incluso se produjo un cambio en el perfil del abstencionista. Si las estimaciones pasadas se realizaban teniendo en cuenta un perfil estable del no votante, las nuevas opciones electorales afectaron a este sector del electorado, que se reparte de forma asimétrica entre las distintas opciones políticas, a la par que personas que tradicionalmente habían votado apuestan por la abstención.

Los cambios producidos en el sistema de partidos generan a su vez nuevas situaciones. Por primera vez en la historia reciente de la democracia española, la Cámara Baja fue incapaz de formar gobierno y se repitieron los comicios el 26 de junio de 2016. Este nuevo escenario rompe la linealidad del sistema, motiva una mayor cobertura mediática, a la par que dificulta la tarea del analista político que nunca se había enfrentado a una repetición electoral. Aún más si se tiene en cuenta que el sistema electoral español implica que pequeños cambios en el voto generan un Parlamento diferente.

Para dificultar aún más la ecuación, es preciso tener en cuenta que la mayor oferta electoral y la desafección política retrasan cada vez más la decisión del voto. Según el Centro de Investigaciones Sociológicas, el $27 \%$ de los electores decidió su voto en la última semana de campaña electoral, unos días antes de las elecciones 0 incluso el mismo día (CIS, 2016). Pese a los citados cambios y al retraso en la decisión del voto, la ley electoral vigente permanece inalterada desde hace más de 30 años. En virtud de la misma, las estimaciones electorales no incorporan la decisión de voto tomada en la última semana de campaña, puesto que está prohibida la publicación de encuestas cinco días antes de los comicios (BOE, 1985). Por ejemplo, la última semana de campaña de las Elecciones Generales de 2015 no se pudo conocer en tiempo real el freno de la fuga de votantes del Partido Popular a Ciudadanos, que motivó que la formación naranja tuviese unos resultados por debajo de los esperados, o el aumento en la intención de voto a Podemos y sus confluencias.

Por otra parte, en las últimas campañas electorales, incluso ha cambiado la vía de comunicación con el electorado. Hemos visto como a los canales tradicionales se ha sumado el uso de redes sociales que conectan especialmente con el electorado más joven (Caldevilla Domínguez, 2009). Si bien su utilización en campaña electoral no es nueva, sí lo ha sido su impacto y centralidad como espacio de competición electoral. Por ello, los principales partidos han apostado fuertemente por las redes sociales. Por ejemplo contratando empresas especializadas en el posicionamiento en Facebook y en el uso de Big Data, como el Partido Popular ${ }^{3}$, o reconociendo públicamente que son las propias redes sociales las que permiten que exista y se difunda el partido, como en el caso de Podemos (Europa Press, 2016).

\section{NUEVOS RETOS PARA LA SOCIOLOGÍA}

El nuevo escenario exige al sector demoscópico una continua adaptación y la necesidad de reinventar sus técnicas y metodología para no perder capacidad explicativa. De forma visual, podríamos describir la tarea principal del sector, como el intento continuo de reducir el espacio entre la realidad social y su medición, en un tiempo de numerosos cambios y situaciones sin precedentes.

Sin embargo, pese a las exigencias de un contexto tan diferente a la anterior legislatura y aún con herramientas sin testar en este nuevo contexto, el sector demoscópico tuvo un alto grado de precisión en las Elecciones Generales de 2015. La Figura 2, evidencia como, a medida que se acercaba la cita electoral, se calibró mucho mejor la intención de voto de los nuevos partidos.

3 El Partido Popular contrató para la campaña de 2016 a la empresa The Messina Group, con amplia experiencia en campañas electorales como la de Obama en su reelección de 2012 o la de David Cameron en 2015. 
- Dos meses antes EÚltima predicción legal =20-D a las 20:00h

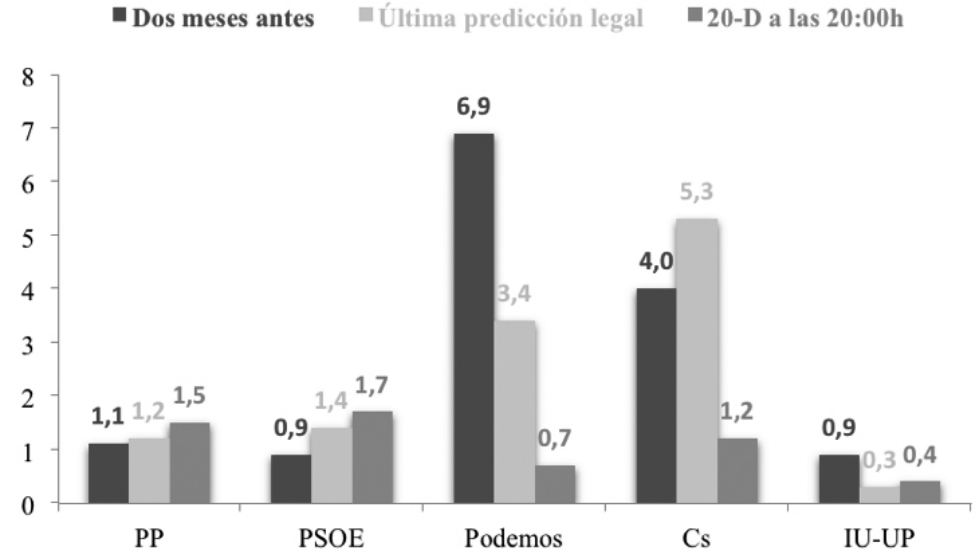

Figura 2. Desviación media por partido del porcentaje de voto (Conjunto de encuestas publicadas). Elecciones Generales $2015^{4}$.

Fuente: Elaboración propia a partir de las encuestas publicadas por cada uno de los 17 institutos que publicaron encuesta con ficha técnica en 2015: dos meses antes de las Elecciones Generales (hasta el 20 de octubre), la última predicción antes de la prohibición legal de publicar estimación de voto y la noche electoral (en el caso de los institutos que dieron estimación el día 20 de diciembre a las 20:00)

Con este grado de acierto, la repetición de los comicios no suponía un desafío equiparable a las Elecciones de 2015, pues se trataba de estimar la intención de voto de los mismos partidos con el recuerdo de voto más reciente de la historia de la democracia: seis meses. En este sentido, se podría teorizar un comportamiento bastante estable. Bien es cierto que la repetición de elecciones ya incluía de por sí un escenario inédito, ¿cómo se comportarían los electores tras la incapacidad del Parlamento para formar Gobierno?

A nivel metodológico, las herramientas de los distintos institutos variaron poco. A tenor del éxito del anterior proceso electoral y con los mismos actores implicados, en GAD3 se mantuvo una metodología muy similar. La recolección de datos se basó en un diseño muestral geográfico formado por 95 zonas de comportamiento electoral homogéneo donde se realizó un tracking de más de 10000 entrevistas, con un cuestionario que no varió sustancialmente durante todo el trabajo de campo y muy similar al de 2015.

Esta metodología nos permitió recopilar un recuerdo de voto muy ajustado al que realmente aconteció en diciembre y durante todo el trabajo de campo se apreciaron tendencias de voto bastante estables. En este sentido, las condiciones parecían las apropiadas para volver a repetir la precisión de los comicios previos.

Por tanto, con un recuerdo de voto tan ajustado al real, cabe centrar el foco en el análisis de los datos como principal causa de la desviación en 2016. La estimación electoral no es un compartimento estanco y el pronóstico de escaños se sustenta en un trabajo mucho mayor de otros indicadores sociales para comprender las tendencias que explican el voto. Por tanto, nos hemos adentrado en un nuevo periodo donde ciertas variables comienzan a ser clave y otras dejan de tener validez y el grado de acierto parte de saber reinventar el análisis.

Por ejemplo, la edad es una variable clave para entender el voto, tanto en España, como en otros paí-

$4 \quad$ La desviación por partido se ha calculado mediante la diferencia (en valor absoluto) del resultado electoral menos la estimación media de los 17 institutos demoscópicos, en cada uno de los tres momentos: dos meses antes de las elecciones, última publicación legal y publicación a las 20:00. 
ses. Son los electores más jóvenes, especialmente afectados por la crisis económica, los que apuestan en mayor medida por los nuevos partidos ${ }^{5}$, por el cam- bio, mientras que el electorado más mayor prefiere a los partidos tradicionales, vinculados a la continuidad del sistema político de los últimos años (Figura 3).

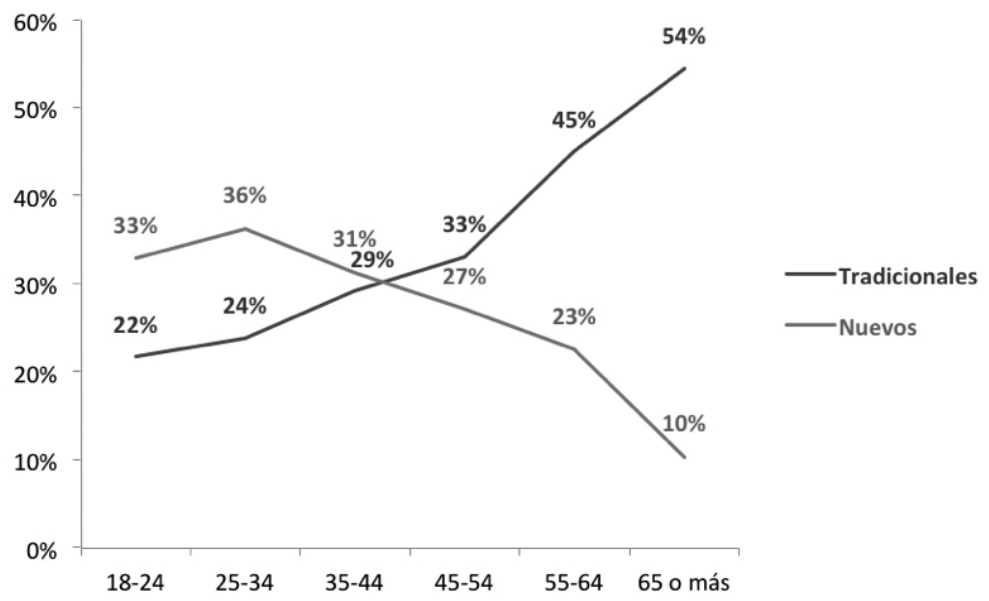

Figura 3. Recuerdo de voto de los cuatro partidos más votados por grupo de edad. Elecciones Generales 2016 Fuente: Elaboración propia a partir de datos del Estudio Postelectoral de las Elecciones Generales de 2016. CIS

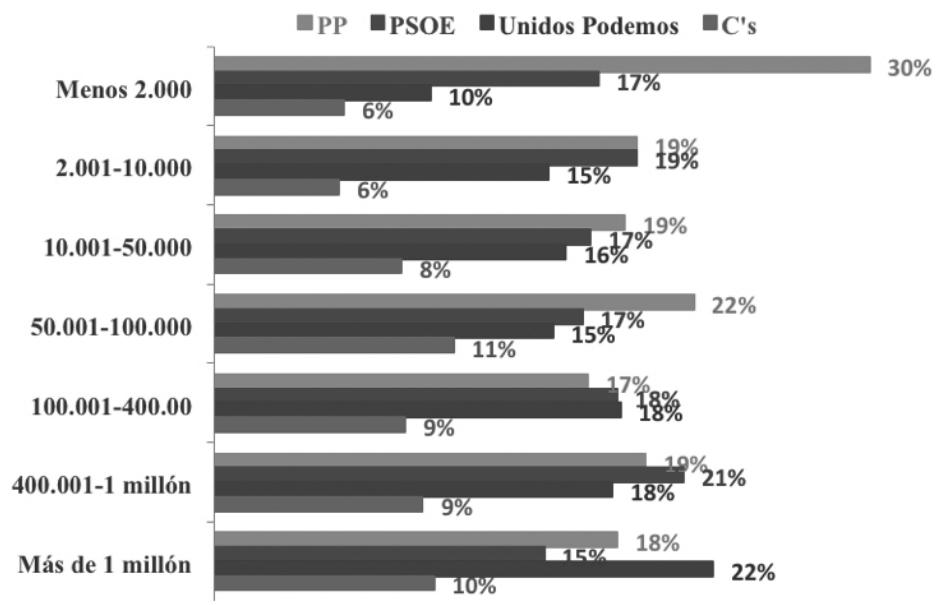

Figura 4. Recuerdo de voto de los cuatro partidos más votados según tamaño del hábitat. Elecciones Generales 2016 Fuente: Elaboración propia a partir de datos del Estudio Postelectoral de las Elecciones Generales de 2016. CIS

5 Partidos tradicionales $=P P+P S O E$, Partidos nuevos=Podemos y sus confluencias+Ciudadanos. 
Tabla 2. Resultados electorales de los partidos con representación parlamentaria de Elecciones Generales 2015-2016

\begin{tabular}{|c|c|c|c|c|}
\hline & \multicolumn{2}{|c|}{2015} & \multicolumn{2}{|c|}{2016} \\
\hline Votantes: & \multicolumn{2}{|c|}{$69,7 \%$} & \multicolumn{2}{|c|}{$66,5 \%$} \\
\hline Abstención: & \multicolumn{2}{|c|}{$30,3 \%$} & \multicolumn{2}{|c|}{$33,5 \%$} \\
\hline Votos válidos: & \multicolumn{2}{|c|}{$99,1 \%$} & \multicolumn{2}{|c|}{$99,1 \%$} \\
\hline Votos nulos: & \multicolumn{2}{|c|}{$0,9 \%$} & \multicolumn{2}{|c|}{$0,9 \%$} \\
\hline Votos a candidaturas: & \multicolumn{2}{|c|}{$99,3 \%$} & \multicolumn{2}{|c|}{$99,3 \%$} \\
\hline Votos en blanco: & \multicolumn{2}{|c|}{$0,7 \%$} & \multicolumn{2}{|c|}{$0,7 \%$} \\
\hline Candidaturas & \% Voto Válido & Diputados & \% Voto Válido & Diputados \\
\hline $\mathrm{PP}$ & $28,7 \%$ & 123 & $33,0 \%$ & 137 \\
\hline PSOE & $22,0 \%$ & 90 & $22,6 \%$ & 85 \\
\hline Podemos/Podemos-IU-Equo & $12,7 \%$ & 42 & $13,4 \%$ & 45 \\
\hline Ciudadanos & $13,9 \%$ & 40 & $13,1 \%$ & 32 \\
\hline ECP & $3,7 \%$ & 12 & $3,6 \%$ & 12 \\
\hline UP-UPeC & $3,7 \%$ & 2 & - & - \\
\hline Podemos-Compromís-EUPV & $2,7 \%$ & 9 & $2,7 \%$ & 9 \\
\hline ERC-CATSÍ & $2,4 \%$ & 9 & $2,6 \%$ & 9 \\
\hline $\mathrm{CDC} / \mathrm{DL}$ & $2,3 \%$ & 8 & $2,0 \%$ & 8 \\
\hline Podemos-En Marea-Anova-EU & $1,6 \%$ & 6 & $1,4 \%$ & 5 \\
\hline EAJ-PNV & $1,2 \%$ & 6 & $1,2 \%$ & 5 \\
\hline EH Bildu & $0,9 \%$ & 2 & $0,8 \%$ & 2 \\
\hline CCa-PNC & $0,3 \%$ & 1 & $0,3 \%$ & 1 \\
\hline
\end{tabular}

Fuente: Elaboración propia a partir de datos del Ministerio del Interior. http://www.infoelectoral.interior.es/

Otra de las variables clave para entender las diferencias en el voto es el tamaño del hábitat, como ilustra la Figura 4. Es en los núcleos más urbanos donde Podemos y Ciudadanos han obtenido un mayor caladero de votos, mientras que el bipartidismo aguanta mejor en el ámbito rural. Esta realidad entronca con el proceso del cambio social en sí, que se produce en primera instancia en las ciudades como entorno más proclive a nuevas tendencias y que, progresivamente, recalan en el resto del territorio nacional.

Sin embargo, la influencia de estas variables, aunque determinantes, ya se incorporaron al análisis de 2015, por tanto, la desviación en las predicciones en 2016 estuvo motivada por otros factores.

Probablemente, el principal elemento que motivó el desacierto generalizado fue la bajada res- pecto a las estimaciones de Unidos Podemos, que se calcula que dejó de sumar en torno a 1 millón de votos. Cabe recordar que la mayoría de sondeos daban por hecho el sorpasso al PSOE en votos y había mucho debate sobre si éste también se produciría en escaños.

No obstante, hay que tener en cuenta que mucho electorado socialista que cambió en 2015 su opción de voto a Podemos, retorna al PSOE tras el intento fallido de gobierno de izquierdas. Respecto a 2015, Unidos Podemos perdió más votos en los territorios donde gobierna y en las zonas donde tradicionalmente IU ha tenido más apoyos, como Asturias $^{6}$.

6 En Asturias, el partido de Pablo Iglesias perdió en 6 meses más de 45000 votos y un $6 \%$ en porcentaje de voto. 
Por su parte, el Partido Popular se benefició de la polarización de la campaña e incorporó 700000 nuevos votantes que confiaron en esta fuerza como alternativa a un Gobierno liderado por Pablo Iglesias. Casi la mitad de estos nuevos votos corresponden a ex-votantes populares que en 2015 apostaron por Ciudadanos y que ahora retornan al PP como estrategia de voto útil. La subida de 14 escaños estuvo por encima de las expectativas, pues se pronosticaba un mayor equilibrio entre bloques izquierda-derecha. Prácticamente la misma noche electoral ya se visualizaba que un Gobierno dirigido por Mariano Rajoy era la única opción aritméticamente posible.

Este trasvase de votos al PP, penalizó mucho a Ciudadanos que vio como con una pérdida del $0,9 \%$ de voto redujo en 8 su cuota de escaños. Si tradicionalmente fue Izquierda Unida la fuerza más penalizada por la ley electoral, en esta ocasión fue el partido de Albert Rivera el más perjudicado. Gran parte de este retorno se debe al apoyo de Ciudadanos a la investidura de Pedro Sánchez, que los ex-votantes populares no secundaron.

Con 85 escaños el PSOE obtuvo por segunda vez consecutiva el peor resultado de su historia, pero una victoria relativa: no se produjo el sorpasso, ni siquiera en votos. Pese a la bajada en escaños y con un descenso de tres puntos en la participación, los socialistas incrementaron $0,7 \%$ en voto y se convirtió en un actor clave para la formación de gobierno. Sin duda, la repetición de elecciones benefició a los partidos tradicionales y el eje izquierda/ derecha volvió a adquirir protagonismo.

\section{PARTICULARIDADES DEL SECTOR DEMOSCÓPICO}

Tras la esperada cita electoral, comenzó un nuevo periodo en el sector demoscópico protagonizado por el análisis y la revisión de los postulados que condujeron a pronósticos erróneos, algunos de los cuáles ya he sintetizado en el apartado anterior. Como en cualquier otra disciplina científica, el error de un modelo conduce a su revisión y reajuste al nuevo contexto, pero en el sector demoscópico median ciertas características que lo diferencia del resto de áreas de la Sociología.
En primera instancia, sorprende la velocidad con la que sucede el proceso entre el trabajo de campo y el análisis. Los distintos cambios sociales suceden de manera relativamente rápida, pero el voto aún más y cambia sustancialmente en pocos días. Por esta razón, la recolección de datos primarios tiene que ser muy cercana al análisis. En este sentido, tenemos que estar testando continuamente la opinión del electorado, teniendo muestra suficiente de varios días para suponer consistencia en los datos y no influencias puntuales del contexto. La necesidad de los sondeos de correr en paralelo a la realidad social se añade un hándicap extra: la prohibición de publicar encuestas la última semana. El juego ha cambiado pero continúa rigiéndose por las mismas reglas. Justo cuando gran parte de los indecisos deciden su voto, la ley electoral española en su artículo 69 prohíbe la publicación de sondeos. Truncar la publicación supone añadir una dificultad extra a la estimación, en tanto que las predicciones no contemplan los decisivos cambios de última hora.

Por otra parte, las encuestas no recogen la opinión pública. Al menos no sólo. Se trata de un proceso circular. Las predicciones electorales también generan opinión pública, en tanto que constituyen un elemento para configurar el voto en función de los equilibrios entre las distintas fuerzas políticas. Esta retroalimentación con la realidad acontece de forma muy rápida y se incorporan a las predicciones casi diariamente, pues en campaña, prácticamente todos los días se están publicando sondeos electorales que se esperan con gran expectación.

Acontece lo que Wert llama la paradoja diabólica, "que explica el que aquellas elecciones en que hay depositada una mayor carga de atención pública tengan más probabilidades de generar en torno a sí una situación de espiral del silencio que desemboque en una estimación desacertada" (Wert, 2002). Sin duda este elemento también constituye un claro desafío y diferencia a la demoscopia del resto de la Sociología. Su exposición pública, muy diferente al resto de áreas, implica que, simplemente observando la realidad social, está influyendo sobre el sujeto de estudio que utiliza la encuesta como herramienta sobre la que basar su estrategia electoral. 
Tras los comicios, el reajuste de los modelos tampoco se hace a puerta cerrada desde la empresa 0 la academia, sino que la exposición pública es notoria. Además, al tratarse de una tarea tan numérica, no sólo el analista evalúa la calidad del modelo, sino que cualquier ciudadano puede cuantificar la desviación. A la dificultad en las predicciones, se le añade que la revisión del análisis y de la metodología para ver su capacidad predictiva la tiene que hacer el investigador a ojos de toda la Sociedad, que exige respuestas de la desviación con la velocidad que caracteriza al sector demoscópico.

En cualquier caso, es preciso ser honestos, reconocer que hacemos ciencia y que el método científico está sujeto, por definición, a revisión y abierto a sucesivas mejoras. Y es precisamente el método lo que hace ciencia a la Sociología y el que dota de garantías a la demoscopia. Es precisamente este conocimiento acumulativo el que nos lleva a incorporar errores y nuevas variables para mejorar el modelo, de forma que se incorpore el cambio social para continuar teniendo una herramienta válida de medición.

Y en este proceso de análisis nos encontramos. Sobre todo porque la predicción electoral sólo es la punta del iceberg de un análisis social más profundo, que se cimenta en conocer las tendencias y cambios sociales analizando otras fuentes de datos. Continuamos analizando los resultados electorales de 2016 para generar nuevos conocimientos que nos ayuden a mejorar el modelo que ya anduvo muy preciso en 2015. En este sentido, estamos obligados a asumir estos nuevos desafíos en un corto periodo de tiempo, dado que las transformaciones sociales ocurren cada vez a mayor velocidad.

\section{REFERENCIAS BIBLIOGRÁFICAS}

BOE. (19 de junio de 1985). Ley Orgánica 5/1985. Ley Orgánica 5/1985, de 19 de junio, del Régimen Electoral General. Madrid: Boletín Oficial del Estado.

BOE. (3 de mayo de 2016). Real Decreto 184/2016. Real Decreto 184/2016, de 3 de mayo, de disoIución del Congreso de los Diputados y del Senado y de convocatoria de elecciones. Madrid: Boletín Oficial del Estado.

Caldevilla Domínguez, D. (2009). Democracia 2.0: La política se introduce en las redes sociales. Pensar la publicidad, 31-48.

Carretero, R. (29 de junio de 2016). Huffington Post. Recuperado el 2 de diciembre de 2016, de Huffington Post: http://www. huffingtonpost.es/2016/06/28/fallosencuestas_n_10715080.html

CIS. (2016). Barómetro postelectoral de las Elecciones Generales de 2015. Madrid: Centro de Investigaciones Sociológicas.

CIS. (2016). Memoria de Actividades 2015. Madrid: Centro de Investigaciones Sociológicas.

Europa Press. (22 de mayo de 2016). eldiario.es. Recuperado el 2 de diciembre de 2016, de eldiario.es: http://www.eldiario.es/politica/ Podemos-delante-sociales-natural-crecieron_0_518648284.html

Rosa, H. (2011). Aceleración social: consecuencias éticas y políticas de una sociedad de alta velocidad desincronizada. Persona y Sociedad, 9-49.

Wert, J. (2002). No le contéis a mi madre que hago encuestas políticas. Ella cree aún que soy pianista en un burdel. REIS, 237-245. 
